\title{
Exploring redox properties of Aromatic Amino Acids in Water: Contrasting single photon vs. resonant multi-photon ionization in aqueous solutions
}

\author{
Anirban Roy, ${ }^{1 \dagger}$ Robert Seidel, ${ }^{1,2}$ Gaurav Kumar $^{1}$ and Stephen E. Bradforth ${ }^{* 1}$ \\ ${ }^{1}$ Department of Chemistry, University of Southern California, Los Angeles, CA-90089-0482, USA \\ ${ }^{2}$ Helmholtz-Zentrum Berlin für Materialien und Energie, Albert-Einstein-Straße 15, 12489 Berlin, Germany
}

*Corresponding author: stephen.bradforth@usc.edu

\begin{abstract}
Direct measurements of the valence ionization energies and the reorganization energies of the three aromatic amino acids, L-Tyrosine, L-Tryptophan, and L-Phenylalanine in aqueous solution using the liquid microjet technique and two different photoemission methods - X-ray photoelectron spectroscopy at $180 \mathrm{eV}$ photon energy and resonant two-photon ionization using $2 \times 267 \mathrm{~nm}$ (4.64 eV) UV laser light are reported. L-Tryptophan has the lowest vertical ionization energy, $7.3 \mathrm{eV}$, followed by Tyrosine $(7.8 \mathrm{eV})$ and Phenylalanine ( $8.7 \mathrm{eV})$. Essentially, no variation in recovered orbital energies is observed comparing near threshold ionization to X-ray ionization. Superior sensitivity of the (background-free) R2PI scheme for solutions with very low solute concentration ( $<2$ millimolar) is demonstrated in contrast to the single-photon XPS measurements, which often requires solute concentrations of 0.1 to 1 molar. This higher sensitivity along with chemical selectivity of the R2PI technique can be exploited for both spectroscopic assignment and as an analytical tool. The nature of the adiabatic ionization energy for the three aromatic amino acids has been explored by the R2PI approach and by empirically formulating the correlation between the estimated ionization onset with electronic and nuclear relaxation on the excited state surface. Our results have implications for understanding oneelectron transfer within enzymes and in redox situations where (ir)reversible deprotonation occur such as those manifest in the biochemistry of oxidation damage.
\end{abstract}

\section{Introduction}

\footnotetext{
${ }^{+}$Current address: Anasys Instruments Corp., Santa Barbara, CA 93101, USA
} 
The redox properties of aromatic amino acids, specifically tryptophan and tyrosine, are of broad scientific interest, as the side groups of these amino acids provide the most versatile and ubiquitous redox activity in the functional proteins of living systems.(1-4) For example, both tryptophan and tyrosine are found to play a significant role in the DNA damage repair mechanism by transferring an electron (or both an electron and a proton) to the nucleobase radical cation (or neutral radical) that is the primary product of the oxidative DNA damage. $(5,6)$

Knowledge about equilibrium and nonequilibrium ionization parameters, such as adiabatic and vertical ionization energies of the constituent amino acids, is also important in the study of radiation damage to proteins. Similarly to oxidative damage in DNA, the initial photo-oxidized site in proteins is found to be nonlocal due to hole migration along the peptide backbone,(7) and this can lead to harmful cross-links between amino acid residues far away from the primary oxidation site. The rates of hole transfer processes are often approximated by Marcus theory where the free energy of the electron transfer reaction, and therefore the redox potential of the electron donor/acceptor couple, plays a prominent role. $(8,9)$

The redox properties of tryptophan and tyrosine have been studied extensively; however, there is difficulty interpreting the reported standard reduction (oxidation) potentials.(10-12) One of the major reasons for the experimental disagreement can be attributed to the complex protonation/deprotonation equilibria that change the measured electrode potentials as a function of $\mathrm{pH}$. Often the standard reduction potential ( $\mathrm{E}^{0}$ at $1 \mathrm{M} \mathrm{H}^{+}$or $\mathrm{pH} 0$ ) and measured redox potential at neutral solutions $\left(E_{7}\right.$ at $\left.\mathrm{pH} 7\right)$ do not follow a linear "Nernstian relationship" due to the existence of several acid-base equilibria, i.e., several pKa, contributing over the measured $\mathrm{pH}$ range. In a key paper, Harriman demonstrated this non-Nernstian behavior over the $\mathrm{pH}$ range 2-13;(10) specifically, he observed a switchover in the ordering of the standard reduction potential of tyrosine and tryptophan at $\mathrm{pH}<3$. Recently, Bradforth and co-workers have used an alternative measurement technique, liquid microjet photoelectron spectroscopy, for measuring vertical ionization energies of biomolecules and to estimate spectroscopic redox potentials for the nucleotides and nucleosides in aqueous solutions. $(13,14)$ Being inherently an ultrafast nonequilibrium technique, photoemission measurements circumvent any contribution to the $\mathrm{E}_{0}$ value coming from fast deprotonation reactions and the irreversibility of the electrochemical process unavoidable on the time scale of standard measurement techniques such as cyclic voltammetry. This is a well-known problem for oxidation of many organic systems.(15)

In this report, we have employed two different photoionization techniques, synchrotron-based $X$-ray photoelectron spectroscopy (XPS) and laser-based resonant two-photon ionization photoelectron spectroscopy (R2PI-PES), combined with the liquid microjet technique to measure the ionization energies of the aromatic amino acids in solution. XPS and R2PI-PES differ with respect to several physical parameters such as chemical selectivity and sensitivity, relative 
ionization cross sections, and probing depth. In resonant two-photon ionization, the first photon excites the molecule to the excited state (HOMO $\rightarrow$ LUMO transition) and the second photon subsequently ionizes the molecule from the resonant intermediate state under the same pulse envelope. Therefore, we can predict a priori the chemical site from which ionization will take place based on the linear absorption spectra and choice of the resonant excitation/ionization wavelength.(16) In Figure 1, we explain the chemical selectivity in the case of aromatic amino acids: when the excitation energy is on resonance with the $\pi \rightarrow \pi^{*}$ transition of the aromatic moiety, the ionization takes place only from the electron promoted from the $\pi$ orbital producing a final state with $\pi^{-1}$ configuration; little or no ionization occurs from the amino or carboxylic acid backbone which absorbs at higher energy. On the other hand, in XPS, the high energy radiation indiscriminately ionizes from all valence orbitals, including those of the solvent (and even core orbitals if the photon energy is high enough). The ionization propensities are determined entirely by ionization cross section and, therefore, impart no chemical selectivity to the ionization. The relative ionization cross sections are also very different in R2PI-PES measurements due to the involvement of the intermediate resonant state. The total ionization signal in resonant measurement depends on both absorption and subsequent ionization cross section, whereas, for XPS, the ionization is solely dependent on one-photon ionization cross section. The resonance enhancement in the R2PI-PES process provides superior sensitivity due to minimum contribution from the nonresonant background compared to single photon ionization for dilute solutions. We also note that recent liquid jet photoemission measurements suggest different probing depths of the photoemission technique at different outgoing electron kinetic energies and this factor must be considered too in comparing results from the two ionization schemes presented here.(17-20)

In this report, which is the first to describe our laser-based liquid jet spectrometer, we have illustrated the enhanced sensitivity of the resonant two-photon ionization technique in the case of sparingly soluble I-tyrosine solution.

\section{Experiments}

All X-ray photoelectron spectroscopy measurements were performed at the U41-PGM beamline at the BESSY synchrotron radiation facility in Berlin using $175 \mathrm{eV}$ photon energy. Experimental details of the photoelectron spectrometer and the liquid microjet have been reported elsewhere.(21) Briefly, a liquid jet of $25 \mu \mathrm{m}$ diameter was injected into a vacuum from a fusedsilica nozzle; the jet velocity was approximately $40 \mathrm{~ms}^{-1}$. Photoelectrons are detected parallel to the synchrotron light polarization vector and perpendicular to the flow of the liquid jet. Emitted photoelectrons pass from the main interaction chamber (operating at $10^{-4} \mathrm{mbar}$ ) through a 500 $\mu \mathrm{m}$ diameter orifice to the differentially pumped detector chamber (operating at $2 \times 10^{-6} \mathrm{mbar}$ ) which houses a hemispherical electron energy analyzer equipped with a multichannel detector. 
The small distance of $0.5 \mathrm{~mm}$ between the liquid jet and the orifice assures that a significant fraction of detected electrons has not suffered from inelastic scattering with water gas-phase molecules near the jet surface. $(21,22)$ The energy resolution of the U41-PGM beamline was better than $65 \mathrm{meV}$ at $175 \mathrm{eV}$ photon energy used for the valence PE measurements, and the energy resolution of the hemispherical analyzer, $\sim 100 \mathrm{meV}$ at $10 \mathrm{eV}$ pass energy, was constant with kinetic energy.

Liquid microjet resonant two-photon ionization experiments were performed at our lab at the University of Southern California (USC) using femtosecond deep ultraviolet (DUV) pulsed excitation. The liquid microjet employed is very similar to the one used at BESSY. The $267 \mathrm{~nm}$ DUV pulse was generated by sum-frequency mixing of the fundamental ( $800 \mathrm{~nm}, 30 \mathrm{fs}$ ) from a Ti:sapphire amplifier system (Coherent Legend Elite, repetition rate $1 \mathrm{kHz}$ ) and its second harmonic $(400 \mathrm{~nm})$ in a $100 \mu \mathrm{m}$ thick type II BBO crystal. The pulse width of the ultraviolet was estimated to be $\sim 200 \mathrm{fs}$ from two-photon absorption in a $1 \mathrm{~mm}$ thick quartz film. $(23,24)$ The polarization of $267 \mathrm{~nm}$ used in the experiment was vertical with respect to the laboratory frame and perpendicular to the time-of-flight axis (orthogonal polarization geometry with respect to the synchrotron experiment). The spot size of the beam at the focus was estimated to be not more than $80 \mu \mathrm{m}$. In our home-built photoelectron spectrometer, we have implemented a magnetic bottle time-of-flight electron detection strategy which enables energy-dispersed detection with significantly higher collection efficiency ( $50 \%)$ than field free time-of-flight detection.(25-27) Photoelectrons were detected at the end of a $50 \mathrm{~cm}$ flight tube using a pair of $40 \mathrm{~mm}$ diameter microchannel plate (MCP) detectors in a chevron configuration (Beam Imaging Solutions). The signal from the anode of the MCP stack was capacitively coupled out and amplified with a 100x gain preamplifier (Phillips Scientific, model 6954B-100) and digitized with a high-speed digitizer card ( $1 \mathrm{GHz}$, DynamicSignals LLC) so that multiple electrons per shot can be resolved and recorded. In all of the experiments, the count rate was maintained to $<10$ electrons/pulse by varying the DUV laser pulse radiant fluence between $1.5 \times 10^{-4}$ and $2.5 \times$ $10^{-3} \mathrm{~J} / \mathrm{cm}^{2}$. The detection chamber was pumped down to $\sim 1 \times 10^{-6} \mathrm{mbar}$ using two turbomolecular pumps (each $300 \mathrm{l} / \mathrm{s}$, Pfeiffer Vacuum) during operation. The source chamber was maintained to $2 \times 10^{-4}$ mbar pressure by using liquid nitrogen cryo traps and a turbomolecular pump (1500 I/s, Pfeiffer Vacuum).

The spectrometer was calibrated using vibrationally resolved three-photon photoemission spectra of water vapor with $267 \mathrm{~nm}$ pulses. The relative energy resolution of the spectrometer $(\Delta E / E)$ is $\sim 10 \%$ at $1.26 \mathrm{eV}$ kinetic energy determined from water vapor photoemission spectra (see Figure S1). We note that the spectrometer energy resolution is a convolution of kinetic energy spread due to flight time distribution, spectral bandwidth of the excitation/ionization pulse, and time response of the detection electronics. The last two contributions are constant, 
while the energy resolution due to flight time distribution varies with the square root of the electron kinetic energy,(25) i.e., higher energy resolution at lower kinetic energy.

Aqueous solutions of I-phenylalanine $(100 \mathrm{mM})$, I-tryptophan $(40 \mathrm{mM})$, I-tyrosine $(1.5 \mathrm{mM})$, cytidine (10 mM - R2PI/0.7 M - XPS), deoxyguanosine monophosphate (5 mM - R2PI/1 M - XPS), and adenosine/adenosine $5^{\prime}$ monophosphate disodium salt (5 mM - R2PI/1 M - XPS) (SigmaAldrich) were prepared without further purification. I-Tyrosine solution was stirred for $5 \mathrm{~h}$ at room temperature for better dissolution. The high concentration nucleotide/nucleoside solutions are achieved by supersaturation. In all of the experiments, sodium chloride/fluoride salts are added ( $\sim 20 \mathrm{mM})$ to minimize the streaming potential.(21,22,28-32) Prepared solutions

were injected into the vacuum chamber using an HPLC solvent delivery pump (Shimazdu) with a constant flow rate of $0.5 \mathrm{~mL} / \mathrm{min}$ and a backing pressure of 4-5 bar. The solution reservoir was kept at $25{ }^{\circ} \mathrm{C}$ in all of the experiments; however, the solution temperature at the interaction region is expected to be lower due to fast evaporative cooling inside the vacuum chamber.(21) The jet thickness was $\sim 20 \mu \mathrm{m}$ based on the liquid-jet nozzle diameter.

\section{Results and Discussion}

X-ray photoemission (top row) and resonant two-photon ionization (bottom row) spectra of the aromatic amino acids are shown in Figure 2. The XPS spectra reported here are derived by carefully subtracting the solvent reference spectrum under identical experimental conditions (i.e., the same salt concentration as well as the same jet temperature and flow rate). Raw spectra were energy calibrated against the water $1 b_{1}$ binding energy $(11.31 \mathrm{eV})(32)$ and intensity normalized against the water $1 b_{2}$ peak and the high binding energy background of water (Figure S2 in the Supporting Information). R2PI-PES measurements, on the other hand, are background free, as the excitation energy $(4.64 \mathrm{eV})$ is only resonant with the $\pi \rightarrow \pi^{*}$ transition and is several eV lower than the water absorption band edge.(33)

Photoemission bands are fitted with a Gaussian or sum of Gaussian functions. The band centers of the fitting functions are assigned as the vertical ionization energies and are listed in Table 1. Tryptophan has the lowest $(7.3 \mathrm{eV})$, and phenylalanine has the highest vertical ionization energy $(8.7 \mathrm{eV})$ for the aromatic amino acid family. We find the vertical ionization energies are almost identical in XPS and R2PI-PES measurements, which primarily suggests the absence of any ultrafast dynamics in the intermediate resonance state accessed in the two-photon experiment (see below). 
In XPS measurements, several higher binding energy photoemission bands are apparent, but the available photon energy in the resonant photoionization experiment $(2 \times 4.64 \mathrm{eV}=9.28 \mathrm{eV})$ limits the range of detection to the lowest one or two ionization channels. The sharp falling edge in the higher binding energy of the R2PI-PES spectra reflects the detector transmission function at low electron kinetic energy ( $0.5 \mathrm{eV})$ and causes greater uncertainty of higher binding energy peak positions when fitted with a Gaussian function. The overall error bar in the binding energy, however, reflects both the uncertainty in calibration of the electron kinetic energy $( \pm 0.14 \mathrm{eV})$ and the streaming potential stemming from electrokinetic charging (estimated $<0.05 \mathrm{eV}$ under our experimental condition).(30) Now, combining all of the above error sources, the convoluted error bar for electron binding energy is estimated to be $\pm 0.15 \mathrm{eV}$. We note that the $\sim 10 \%$ relative energy resolution $(\triangle E / E)$ of the instrument has a minimal effect in determining the position of peak maxima (VIE), since the typical photoemission peak width in liquids is $\sim 1 \mathrm{eV}$.

Due to the lower solubility of I-tyrosine in water ( $\sim 2 \mathrm{mM})$, it was impossible to obtain an X-ray photoelectron spectrum with an acceptable signal-to-noise ratio ( $\mathrm{S} / \mathrm{N}$ ) even after a longer acquisition time (Figure 2c). However, the resonance enhancement in the R2PI-PES measurement yields PE spectra with excellent $\mathrm{S} / \mathrm{N}$ and comparable to the other amino acids (Figure $2 \mathrm{f}$ ). This clearly illustrates the superior sensitivity of the R2PI-PES measurements over single photon ionization techniques for samples at low concentration.

There is an extensive literature on the gas phase photoemission of isolated amino acids. Previous works by Ham,(34) Campbell,(35,36) Inokuchi,(37) and Prince(38) reported vertical ionization energies for isolated aromatic amino acids. These experimental results along with theoretical calculations(39) facilitated the spectral assignments of the gas phase photoemission bands. The peaks between 8 and $11 \mathrm{eV}$ were assigned to ionization from the $\pi$-orbitals centered on the aromatic moiety (using the notation of ref $(35), \pi_{3}$ : HOMO, $\pi_{2}$ : HOMO-1) and the nonbonding orbital of the amine nitrogen $\left(n_{N}\right)$ in the amino acid residue.(34-37) Comparing our solution Xray photoemission spectra against the gas phase results, we can assign the XPS bands as indicated in Table 1. The energetics of the ionization process is expected to be affected by the introduction of a highly polar environment when transitioning from gas phase to aqueous solution. However, the extent of the solvent influence on the VIE will depend strongly on the relative stabilization of the initial state (neutral) and the final state (radical cation) in water. $(40,41)$ For example, we observe the solvation shift in the vertical ionization energy is greater for the polar chromophores tryptophan and tyrosine as compared to the nonpolar chromophore in phenylalanine.

Assigning the R2PI-PES band, on the other hand, is conceptually easier, since, in the one-electron picture, the resonant photoionization must take place solely from the orbital promoted in the optical excitation. In Figure 3, we present the UV/Vis absorption spectra of the aromatic amino acids and their aromatic side chain precursors (benzene, indole, and phenol). Evidently, at our 
excitation wavelength ( $267 \mathrm{~nm}$ ), we only excite the aromatic moieties in the amino acids ( $\pi \rightarrow \pi^{*}$ transition). Hence, the resonant photoemission spectra is attributed to ionization from occupied $\pi$ orbitals and confirms our XPS assignments based on the gas phase literature discussed above.

We note that for tryptophan and tyrosine we observe two resonant photoemission bands (see Figure 2e) which we assign to ionization from $\mathrm{HOMO}\left(\pi_{3}\right)$ and HOMO-1 $\left(\pi_{2}\right)$ orbitals. This might seem quite counterintuitive, since electronic excitation primarily involves HOMO $\rightarrow$ LUMO transition; therefore, subsequent ionization should only produce final states with $\pi_{3}^{-1}$ configuration. In the case of tryptophan, two overlapping transitions $S_{0} \rightarrow \pi \pi^{*}\left({ }^{1} L_{a}\right)$ and $S_{0} \rightarrow \pi \pi^{*}$ $\left({ }^{1} L_{b}\right)$ (based on Platt-Murrell's nomenclature for aromatic molecules) $(42,43)$ constitute the electronic absorption band between 240 and $300 \mathrm{nm(44)}$ (cf. Figure 3). Roos and co-workers have shown that the major contribution to the above two transitions correspond to HOMO $\rightarrow$ LUMO (54\%) and HOMO-1 $\rightarrow$ LUMO (44\%) transitions, respectively.(45) Therefore, we can argue that the admixture of those transitions at our resonant photoionization wavelength leads to both $\pi_{3}{ }^{-}$ 1 and $\pi_{2}{ }^{-1}$ electronic configuration final states. However, this argument does not apply to tyrosine, since the upper $\pi \pi^{*}$ state is too high in energy to be excited with a $267 \mathrm{~nm}$ pump.(46) In resonant multiphoton ionization of isolated phenol, Weber and co-workers observed photoelectron bands due to configuration interaction.(47) They estimated $12 \%$ contribution to the total photoelectron signal from configuration interaction when excited to the $S_{1}$ state intermediate. We have also performed R2PI-PES measurements on aqueous phenol (see Figure S3 in the Supporting Information), and a comparison with the reported X-ray photoemission spectra(13) reveals that the excited state configuration interaction also contributes in the condensed phase. The presence of the $\pi_{2}{ }^{-1}$ configuration in the final states for both tryptophan and tyrosine hence indicates significant configuration interaction in the intermediate excited state. To the best of our knowledge, this is the first time the experimental evidence of configuration mixing has been presented in the condensed phase using photoemission spectroscopy.

The adiabatic ionization energy (AIE) can also be estimated from the onset of the photoelectron spectra as listed in Table 2. The onset is defined as the intersection of the noise level and the rising edge of the PE spectra when plotted in the logarithmic scale at the intensity axis.(48) The rising edge has been defined by the slope line drawn at $1 / e^{2}$ value of the lowest binding energy peak intensity (red line in Figure 4). The noise level, on the other hand, is an ill-defined quantity and depends on the instrument sensitivity at the low signal intensity. We have noticed that the resonant photoemission measurements offer a greater dynamic range in electron counts (>2000:1) than the reference-subtracted spectra in X-ray photoemission measurements (<400:1). This is because we are not subtracting solvent background signals and their associated noise in the R2PI-PES technique; this determines the baseline level at low binding energy (cf. Figure 4). This particular advantage allows us to better assign the noise floor while extracting the ionization 
onset in the resonant photoemission measurements. Therefore, we use our R2PI-PES results to estimate the adiabatic ionization energy. We note that a range of estimates for the baseline (see Figure 4) can be assigned in each of the R2PI-PES spectra; this provides an additional uncertainty in the threshold (from \pm 0.05 to $\pm 0.1 \mathrm{eV}$ depending on data set). When combined with uncertainties in the binding energy scale $( \pm 0.15 \mathrm{eV})$, this results in an overall $\leq \pm 0.18$ eV error in the estimated ionization threshold. Differences in the shape of the rising edge between the XPS and the R2PI measurement of tryptophan can be explained by different ionization cross sections, by the water background present in the XPS, and, as explained below, by the finite lifetime of the laser pulses in R2PI. The reorganization energy, which is defined by the difference between the vertical and adiabatic ionization energy, is found to be $\sim 1.2-1.5 \mathrm{eV}$ (Table 2), which is within the acceptable range of values for biomolecules with similar aromatic moieties, $(13,14)$ therefore suggesting that the AIE values extracted in this way are reasonable.

The gas phase adiabatic ionization energy is rigorously the energy difference between the neutral (initial) and cationic (final) states in their respective vibrational ground states [AIE $=E$ (cation, $v^{\prime \prime}$ $=0)-E\left(\right.$ neutral, $\left.\left.v^{\prime}=0\right)\right]$. In solution, we need to consider solvent modes and the finite temperature. A common description is to define the aqueous-phase AIE as the energy difference between the equilibrated structures of the neutral and ionized molecule (with its corresponding relaxed solvent structure) with a vacuum electron. $(49,50)$ Following linear response theory, the collective molecular and solvent motion lead to parabolic free energy surfaces (Marcus parabolas) in a one-electron transfer reaction.(8) Now, the energy difference between two parabolas at their respective equilibrium solvent configurations represents the change in free energy due to oxidation $\left(\Delta G^{0}\right)$ and is therefore intrinsically related with the standard one-electron oxidation potential $\left(E^{0} ; \Delta G^{0}=-n F E^{0}\right)$. Hence, we can compare our estimated AIE values with the reported $E^{0}$ values, referenced to the standard hydrogen electrode (SHE), from the electrochemistry literature.

The equilibrium redox reaction in water under cyclic voltammetry conditions involves both electron and proton transfer as shown below

$$
\begin{gathered}
\operatorname{Trp}(-\mathrm{H})^{*}+\mathrm{H}^{+}+\mathrm{e}^{-} \Leftrightarrow \operatorname{Trp}(1 \mathrm{a}) \\
\operatorname{Tyr}(-\mathrm{H})^{*}+\mathrm{H}^{+}+\mathrm{e}^{-} \Leftrightarrow \operatorname{Tyr}(1 \mathrm{~b}) \\
E=E^{0}\left(\mathrm{~A}(-\mathrm{H})^{*}, \mathrm{H}^{+} / \mathrm{A}\right)+\frac{R T}{F} \ln \left(K_{\mathrm{r} 1} \cdot \frac{\left[\mathrm{H}^{+}\right]+K_{\mathrm{n} 1}}{K_{\mathrm{r} 1}+\left[\mathrm{H}^{+}\right]}\right)+\frac{R T}{F} \ln \frac{\left[\mathrm{A}^{*}\right]_{\text {total }}}{[\mathrm{A}]_{\text {total }}} \text { (1c) }
\end{gathered}
$$

where $R$ is the gas constant and the acid dissociation constants $K_{\mathrm{r} 1}$ refer to equilibria $\mathrm{A}^{+\bullet} \Leftrightarrow \mathrm{A}+$ $\mathrm{H}^{+}$and $K_{\mathrm{a} 1}$ to $\mathrm{A} \Leftrightarrow \mathrm{A}^{-}+\mathrm{H}^{+}$, where $A \equiv \operatorname{Trp}$ and Tyr.(14) The last term containing the ratio of total 
concentrations is frequently omitted in the literature, because midpoint potentials are usually reported, where this ratio is equal to unity.

The quantity of interest, $\Delta G^{0}$, for the one-electron redox reaction without follow up reactions is best achieved via the photoionization process, which yields the instantaneous distribution of the oxidized species, before the slower proton transfer steps. The relevant reactions can be expressed as $\underline{(14)}$

$$
\begin{gathered}
\operatorname{Trp}^{+\bullet}+\mathrm{e}^{-} \rightarrow \operatorname{Trp}(2 \mathrm{a}) \\
\operatorname{Tyr}^{+\bullet}+\mathrm{e}^{-} \rightarrow \operatorname{Tyr}(2 \mathrm{~b}) \\
E=E^{0}\left(\mathrm{~A}^{*+} / \mathrm{A}\right)+\frac{R T}{F} \ln \left(\frac{\left[\mathrm{H}^{+}\right]+K_{\mathrm{al}}}{K_{\mathrm{r} 1}+\left[\mathrm{H}^{+}\right]}\right)+\frac{R T}{F} \ln \frac{\left[\mathrm{A}^{*}\right]_{\text {total }}}{[\mathrm{A}]_{\text {total }}}(2 \mathrm{C})
\end{gathered}
$$

Harriman reported the oxidation potentials of tyrosine and tryptophan in water using cyclic voltammetry and illustrated the variation of derived midpoint potential values with $\mathrm{pH}$ as expected by eq 1c or $\underline{2 c}$ (see Table 2). Interestingly, the potentials for tyrosine and tryptophan indeed behave differently at $\mathrm{pH}$ values lower than 4.5; the potential for tyrosine increases almost linearly with decreasing $\mathrm{pH}$, while tryptophan maintains a constant potential, resulting in a reversal in ordering of the $E^{0}$ beyond $\mathrm{pH} 3$ (see Figure 2 in ref (10)). This is due to the very different $\mathrm{p} K_{\mathrm{a}}$ values for the two radical cations: $\mathrm{p} K_{\mathrm{r} 1}=4.7$ for $\operatorname{Trp}^{+\bullet}$ (from ref $\underline{(10)}$ ) whereas $\mathrm{p} K_{\mathrm{r} 1} \sim-2.75$ for $\mathrm{Tyr}^{+\bullet}$, using the literature value for phenol.(72) Using the midwave potentials at $\mathrm{pH} 2$ and at $\mathrm{pH} 7$ and eq $2 \mathrm{c}$, we can estimate that $E^{0}\left(\mathrm{Tyr}^{+\bullet}, \mathrm{Tyr}\right)$ will be $\sim 0.4 \mathrm{~V}$ higher than $E^{0}\left(\operatorname{Trp}^{+\bullet}, \operatorname{Trp}\right)$, the same order observed in the photoionization experiment with approximately the same difference in $E^{0}$ (Table 2). However, the absolute values for $E^{0}\left(\operatorname{Tyr}^{+\bullet}, \operatorname{Tyr}\right)$ and $E^{0}\left(\operatorname{Trp}^{+\bullet}, \operatorname{Trp}\right)$ are both about $0.5 \mathrm{~V}$ higher via the photoemission technique. In recent work, Signorell and Suzuki(78) have shown that inelastic scattering of the outgoing electron plays an important role on the PE peak shape and in an electron kinetic energy dependent fashion; correction due to inelastic scattering is essential to extract correct binding energies. $(78,79)$ If we consider the eKE at peak for the first ionization transition of each amino acid in Figure 4 , the computed peak shifts from inelastic scattering simulations at equivalent eKEs from ref $(78)$ are $\sim 0$ for phenylalanine, $0.4 \mathrm{eV}$ for tyrosine, and $\sim 0.5 \mathrm{eV}$ for tryptophan, all to lower binding energy and thus lower $E^{0}$. Table 3 summarizes the extrapolated $E^{0}\left(\operatorname{Tyr}^{+\bullet}, \operatorname{Tyr}\right)$ and $E^{0}\left(\operatorname{Trp}^{+\bullet}, \operatorname{Trp}\right)$ on the basis of resonant photoemission and from electrochemical measurements.

To evaluate further the resonant photoemission technique for $E^{0}$ estimation, we performed R2PIPES experiments on the following nucleosides/nucleotides: cytidine (Cyt), adenosine (Ado), and deoxyguanosine monophosphate (dGMP) in water where the $E^{0}\left(A^{+\bullet}, A, A \equiv\right.$ nucleobase) have been subject to a more detailed study, in particular by electrochemical measurements in aprotic 
solvents. In a recent work, Schroeder et al. reported the XPS measurements of these DNA components and derived the standard one-electron oxidation potentials in aqueous solutions.(14) The authors addressed the subtlety in calculating standard state $E^{0}$ values by carefully considering all possible acid-base equilibria of the reduced and oxidized form of the redox couple and illustrated the deviation from the $E^{0}$ values reported earlier in the literature $(52)$ (Table 4 in ref (14)). Most importantly, they established that $E^{0}$ values derived from VIEs from photoelectron spectroscopy and computed reorganization energies within a polarized continuum model for the solvent were in good agreement with electrochemical $E^{0}$ values measured in the aprotic solvent acetonitrile.

We here compare $E^{0}$ values derived from R2PI-PES of the nucleosides/nucleotide (Figure 5 ) when referenced to SHE $\left(E^{0}=\mathrm{AIE}-E^{0}\left(\mathrm{H}^{+} / 1 / 2 \mathrm{H}_{2} \mid \mathrm{Pt}\right)\right.$, where $\left.E^{0}\left(\mathrm{H}^{+} / 1 /{ }_{2} \mathrm{H}_{2} \mid \mathrm{Pt}\right)=4.28 \mathrm{~V}(13,53,54)\right)$ with those reported in ref $(14)$ in Table 2 . We notice that the values extracted from the R2PI-PES measurements in all cases including adenosine overestimate both the $E^{0}$ reported in Schroeder et al.,(14) as well as the reported $E^{0}$ in acetonitrile,(54) in the worst case by $>0.8 \mathrm{eV}$ for dGMP, a case for which past electrochemical (52) and XPS estimates for $E^{0}$ are in close agreement. This is somewhat larger than can be accounted for by a $0.4-0.5 \mathrm{eV}$ correction due to inelastic scattering to the PE peak shape described above. Furthermore, directly comparing the R2PI with XPS spectra in Figure 5 suggests a much poorer match in the first ionization peak onset and peak shape; the alignment is much poorer than we see in Figure 4 for amino acids.

In order to explain this disparity in the photoemission behavior and why the situation differs so much between amino acids and nucleobases, we need a closer look into the physical picture of the R2PI-PES process. For the nonresonant photoionization process, AIE is strictly pulse-widthindependent, since there is no stationary intermediate state. In an R2PI process, the initial excitation to the resonant state and the subsequent ionization take place within the same optical pulse envelope; the intensity-dependent ionization rate is kinetically competing with electronic and nuclear relaxation from the initially excited state. Therefore, the observed vertical ionization energy (VIE) as well as the ionization onset/threshold (AIE) may manifest pulse-width-dependent (and intensity-dependent) character. $(73,74)$ We summarize these competing scenarios in Figure $\underline{6}$. The variation of the observed ionization onset with laser pulse width can be empirically expressed via eq 3 .

$$
\operatorname{AIE}(\Delta t)=\operatorname{AIE}(0)+\frac{R_{\text {relhx }}}{R_{\text {relax }}+R_{\text {ion }}}\left[\Delta E\left(1-\mathrm{e}^{-\Delta t / t_{\text {ralr }}}\right)\right]
$$

Here $\operatorname{AIE}(\Delta t)$ and $\operatorname{AIE}(0)$ represent pulse-width-dependent and intrinsic values for the ionization onsets, respectively. $\Delta t, \tau_{\text {relax, }}$ and $\Delta E$ denote laser pulse width, relaxation time, and relaxation energy, respectively. $R_{\text {relax }}$ and $R_{\text {ion }}$ express the relaxation and resonant two-photon ionization 
rates. Note that $R_{\text {relax }}$ and $R_{\text {ion }}$ can be expressed as usual in terms of respective rate constants, the laser intensity $(I)$, and the excited state population as follows.

$$
\begin{aligned}
& R_{\text {ion }}=k_{\text {ion }} \cdot I \cdot[\text { no. of excited molecules }] \sim k_{\text {ion }} \cdot I^{2}(4 \mathrm{a}) \\
& R_{\text {relax }}=k_{\text {relax }} \cdot[\text { no. of excited molecules }] \sim k_{\text {relax }} \cdot I(4 \mathrm{~b})
\end{aligned}
$$

where $k_{\text {relax }}=\tau_{\text {relax }}{ }^{-1}$. The quadratic dependence of laser pulse intensity on the R2PI process is illustrated in Figure S6 in the Supporting Information.

When ionization dominates over electronic or nuclear relaxation ( $R_{\text {ion }} \gg R_{\text {relax }}$ ), the majority of the excited molecules ionize from the Franck-Condon region and the shift due to relaxation (the exponential term) is irrelevant. Therefore, the observed R2PI ionization onset would be the same as in the case of nonresonant ionization (scenario 1 in Figure 6).

On the contrary, when $R_{\text {ion }} \ll R_{\text {relax }}$ a more dynamical character to the AIE might be expected. First, in the delta pulse excitation limit (or infinitely long nuclear/electronic relaxation time), $\Delta t / \tau_{\text {relax }} \rightarrow 0$, and we would again expect to see no pulse width dependence as the exponential term goes to unity (scenario $2 \mathrm{a}$ in Figure 6 ). With a shrinking pulse width, the peak intensity also increases, therefore further favoring the rate of ionization over relaxation. This effect combined with vanishing of the relaxation term $\left[\Delta E\left(1-\exp \left(-\Delta t / \tau_{\text {relax }}\right)\right)\right]$ at the short pulse limit again strictly favors the intrinsic ionization onset.

However, for finite pulse width experiments (or when $\Delta t$ and $\tau_{\text {relax }}$ are of similar magnitude), there will be competition between instantaneous ionization from the Franck-Condon region of the $S_{n}$ surface and nuclear/electronic relaxation followed by ionization from the relaxed electronic state, weighted by their respective rates (scenario $2 \mathrm{~b}$ in Figure 6 ). Depending on the magnitude of $\Delta t / \tau_{\text {relax, }}$ this would affect both the peak maximum (VIE) as well as the slope of the rising edge of the PE signal (red line in Figures 4 and $\underline{5}$ ), thus altering the estimated AIE values as discussed below.

We can now examine whether the above physical picture is consistent with the experimental results. Noting the empirical nature of eq 3 , we emphasize that only a qualitative comparison may be drawn. With this precaution, we can examine a test case for adenosine (Ado). From gasphase time-resolved photoemission studies in cold molecular beams, Stolow and co-workers found that the excited state dynamics of an isolated adenine can be fit using three exponential decays with time scales $<50 \mathrm{fs}, 750 \mathrm{fs}$, and several nanoseconds. $(55,56)$ In more recent gas phase experiments at room temperature, Chatterley et al. reported similar relaxation dynamics of $\mathrm{dAMP}^{-}$with time constants $<60 \mathrm{fs}$ and $\sim 300 \mathrm{fs}$. (75) The sub-100 fs decay is assigned as ultrafast relaxation from optically bright $\pi \pi^{*}$ to the close-lying dark $n \pi^{*}$ state; however, the exact nature of the relaxation pathway is still debated due to possible intermixing of the two states along the 
decay pathway. In aqueous solution, similar ultrafast dynamics has been observed in dispersed transient absorption measurements.(57) In the condensed phase, the fastest relaxation time scale of $55 \mathrm{fs}$ has been attributed to rapid intersystem crossing between two close-lying $\pi \pi^{*}$ states $\left({ }^{1} \mathrm{~L}_{b} \rightarrow{ }^{1} \mathrm{~L}_{a}\right)$. Regardless of the nature of the final electronic state, both in gas and in solution, adenine undergoes rapid relaxation with a time scale on the order of our excitation pulse width $(200 \mathrm{fs})$. With the assumption of predominant nuclear/electronic relaxation $\left(R_{\text {ion }} \ll R_{\text {relax }}\right)$, we can use $\operatorname{AIE}(\Delta t)=6.8 \mathrm{eV}$, estimated from our R2PI-PES experiment (Table 2); $\operatorname{AIE}(0)=6.38 \mathrm{eV}$, derived from $E^{0}$ values in ref $(14) ; \tau_{\text {relax }} \sim 50 \mathrm{fs}$ and $\Delta t \sim 200 \mathrm{fs}$, to calculate the relaxation energy $(\Delta E) \sim$ $0.4 \mathrm{eV}$. This value is in reasonable agreement, particularly when acknowledging the possibility for an extra correction for inelastic scattering, to the calculated energy difference of $0.36 \mathrm{eV}$ between the two close-lying $\pi \pi^{*}$ states $\left({ }^{1} \mathrm{~L}_{a}\right.$ and $\left.{ }^{1} \mathrm{~L}_{b}\right)$ in water. $(58) \mathrm{A}$ similar explanation applies to dGMP and cytidine where ultrafast electronic and nuclear relaxation from the initially excited state can account for the mismatch between one-photon and resonant two-photon ionization onset values. $(59-61,76,77,80)$ These results corroborate our physical picture of the pulse-widthdependent photoionization and elucidate the difference in the $E^{0}$ values emerging from R2PI-PES measurements and those derived from X-ray photoemission measurements.(7)

We now consider how this picture translates to the case of amino acids. The initial excited state dynamics of the aromatic amino acids are expected to resemble that of their model aromatic precursors. The excited state photophysics of aqueous tryptophan and its model chromophore indole has been explored extensively at 266-290 nm excitation using various time-resolved techniques.(62-65) In a fluorescence up-conversion study, Bräm and co-workers found an ultrafast relaxation component with $160 \pm 40 \mathrm{fs}$ time scale $\left(\tau_{\text {relax }}\right)$ and a $720 \pm 110 \mathrm{~cm}^{-1}(\Delta E)$ shift for tryptophan in water, which is attributed to the fast inertial response of the solvent. (62) Using these parameters, we estimate the decrease in the ionization onset within our pulse width (200 fs) to be $0.09 \pm 0.01 \mathrm{eV}$ compared to the nonresonant ionization for tryptophan, well within the error of our AIE estimation $( \pm 0.18 \mathrm{eV})$. In other words, for tryptophan, we should not observe any pulse-width-dependent shift in the AIE.

In recent transient absorption studies, Barry and co-workers observed excited state dynamics of aqueous tyrosine (at $\mathrm{pH}$ 9) at a time scale $>10 \mathrm{ps}$ at $280 \mathrm{~nm}$ excitation.(66) The authors did not observe any sub-picosecond dynamics within their experimental time resolution of $360 \mathrm{fs}$. Similarly, our recent broadband transient absorption studies on aqueous phenol solution at 267 $\mathrm{nm}$ pump (resolution $<50 \mathrm{fs}$ ) do not show any indication of sub-picosecond solvent or vibrational relaxation.(67) The longer ( $\tau_{\text {relax }}>1 \mathrm{ps)}$ ) relaxation time scales observed for tyrosine and its model chromophore phenol are not expected to change in photoionization threshold energy (ionization onset) simply because the $\Delta t / \tau_{\text {relax }}$ factor in eq 3 approaches zero for ultrashort excitation pulses $(\Delta t \sim 200 \mathrm{fs})$. 
In the case of benzene, the aromatic chromophore of phenylalanine, the first excited state has a lifetime in the order of $2 \mathrm{~ns}$ in water when excited close to the $S_{1}$ origin (ca. $\left.260 \mathrm{~nm}\right) .(67,68)$ The experimental fluorescence lifetime does not change when the pump wavelength is reduced to $249 \mathrm{~nm}$ (1773 $\mathrm{cm}^{-1}$ higher than the 0-0 transition).(69) From the absorption spectra of phenylalanine (cf. Figure 3), we can conclude that at $267 \mathrm{~nm}$ we are exciting close to the $0-0$ transition and expect no vibrational relaxation $(\Delta E \sim 0)$. The reported electronic relaxation time scale is also orders of magnitude higher than our pulse width. $(70,71)$ Therefore, the twofold effect of minimal excited state relaxation energy $(\Delta E \sim 0)$ and slow relaxation time $\left(\Delta t / \tau_{\text {relax }} \sim 0\right)$ results in $\operatorname{AIE}(\Delta t)$ being almost identical to $\operatorname{AIE}(0)$ according to eq 3 . Hence, considering this caseby-case evaluation, we conclude that, in cases where there are only minor intermediate state relaxation dynamics, R2PI-PES introduces no additional complications compared to nonresonant PES.

We find that PE spectroscopy in general provides a useful alternative pathway to evaluating standard oxidation potentials for aromatic containing organic molecules with irreversible redox couples, particularly where the radical cation $\mathrm{p} K_{\mathrm{a}}$ is not well established. The precision of extracted oxidation potentials, which are not currently as high as those determined by typical electrochemical methods, are mainly influenced by calibration error and uncertainty in establishing the threshold, $\sim \pm 0.18 \mathrm{eV}$. Both factors can be improved. Importantly, to minimize systematic error in binding energy estimation, a careful consideration of inelastic electron scattering to the PE peak shape is required; this may be achieved either by correction that includes simulation of the effect of inelastic scattering or by optimal choice of photon energy to minimize its impact.(78)

\section{Conclusion}

We have measured the vertical ionization energies of the aromatic amino acids in aqueous solutions using two different photoemission techniques, steady state X-ray and resonant twophoton photoemission. Our results show that tryptophan has the lowest ionization energy followed by tyrosine and phenylalanine. As expected, this observed ordering of primary oxidation propensity for tyrosine and tryptophan differs from electrochemical measurements at $\mathrm{pH} 7$, which necessarily include fast deprotonation steps. An understanding of the half reaction considered, the effect of $\mathrm{pH}$ on the standard reduction potential, and a closer look at the relevant deprotonation energetics explains the different ordering in the $E^{0}\left(A^{+\bullet}, A\right)$ values, and we find consistency between derived $E^{0}\left(\operatorname{Tyr}^{+\bullet}, \operatorname{Tyr}\right)$ and $E^{0}\left(\operatorname{Trp}^{+\bullet}, \operatorname{Trp}\right)$ from extrapolating electrochemical values and corrected PE thresholds (Table 3). 
Interestingly, R2PI-PES and XPS probe very different depths from the solution surface, as the widely varying photon energies involved lead to different kinetic energies of the outgoing photoelectrons. We expect R2PI to primarily probe the bulk ( $<10 \mathrm{eV}$ kinetic energy) while XPS $(>150 \mathrm{eV})$ is essentially probing the surface/interface. We do not observe significant energy shifts here, but this difference between near threshold ionization in R2PI-PES to X-ray ionization may be useful for systems known to have an interface-propensity and where the electronic structure is more sensitive to environment.

We have also demonstrated the superior sensitivity of the resonant two-photon ionization scheme for solutions with low solute concentration in contrast to the single-photon XPS measurements used hitherto and often requiring solute concentrations of $0.1-1 \mathrm{M}$. This combination of higher sensitivity and preferential ionization from a specific chemical moiety makes R2PI-PES a unique tool for spectroscopic assignment and chemical analysis.

The near-instantaneous nature of the photoionization process has been exploited, leading to estimations of the intrinsic standard redox potentials for systems in aqueous solutions that otherwise rapidly deprotonate, on a time scale faster than the establishment of the reversible equilibria required for electrochemical techniques like cyclic voltammetry. Ionization thresholds can be established with greater certainty using R2PI-PES compared to XPS, and for situations where there is no significant intermediate state relaxation dynamics taking place on the time scale of the pulse width, such as in aromatic amino acids, it should be possible to derive reliable standard oxidation potentials $E^{0}\left(A^{+} \bullet / A\right)$. On the other hand, for systems that do exhibit rapid electronic or vibrational dynamics that lead to $>0.1 \mathrm{eV}$ energetic relaxation, we have explored the dynamical nature of the adiabatic ionization energy revealed in the R2PI-PES and empirically formulated the correlation between estimated ionization onset with electronic and nuclear relaxation on the excited state surface. In principle, reliable oxidation energies could be established for such systems from pulse-width-dependent R2PI photoelectron spectra. This would require that intermediate state relaxation does not take place so rapidly that the energetic uncertainty in the pulse spectrum then exceeds the other instrumental factors limiting the electron kinetic energy resolution.

\section{Supporting Information}

The Supporting Information is available free of charge on the ACS Publications website at DOI: 10.1021/acs.jpcb.7b11762.

R2PI time-of-flight spectrometer calibration; subtraction, normalization, and calibration of XPS spectra; XPS and R2PI measurements for aqueous phenol; reorganization energy; extraction of AIE from XPS spectra; intensity dependence of the R2PI signal; and additional references 


\section{AUTHOR INFORMATION}

Corresponding Author

*E-mail: stephen.bradforth@usc.edu.

\section{ORCID}

Stephen E. Bradforth: 0000-0002-6164-3347

\section{Notes}

The authors declare no competing financial interest.

\section{Acknowledgements}

S.E.B. acknowledges support from the National Science Foundation (grant CHE-0957869 and CHE-1301465). R.S thanks the Deutsche Forschungsgemeinschaft for a DFG scholarship to support his postdoctoral work at USC (SE 2253/1-1). The authors also thank the HZB staff for their assistance during the beam times at BESSY II. 


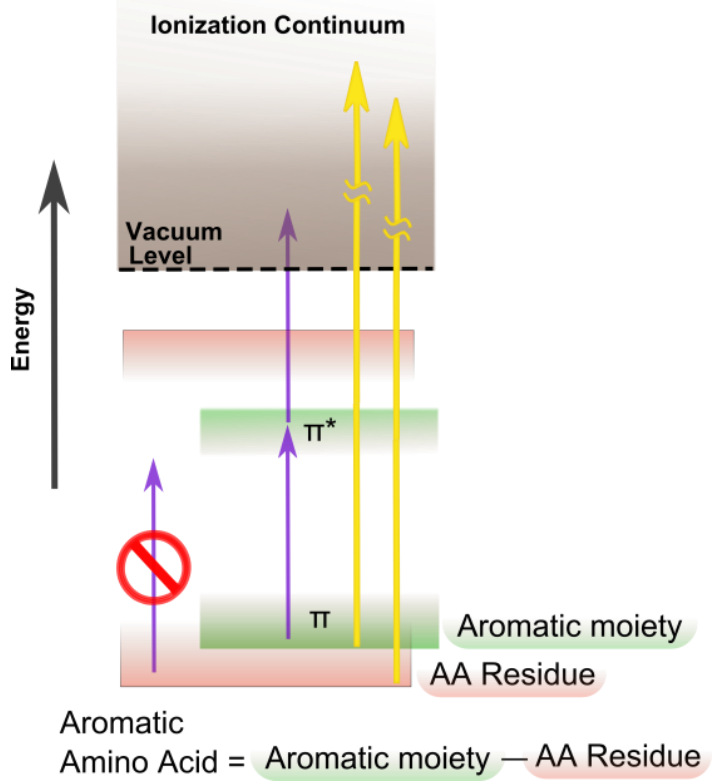

Figure 1: Schematic illustration of the X-ray and resonant two photon ionization photoemission process. High energy X-ray radiation leads to ionization of all the valence orbitals and produces photoelectrons at high kinetic energies (yellow arrows). In contrast, in resonant photoemission, ionization takes place only from those orbitals which are involved in resonant electronic excitation (purple arrows) with the first photon. Since the total energy deposited in the latter process is much smaller, it also yields lower kinetic energy electrons. 

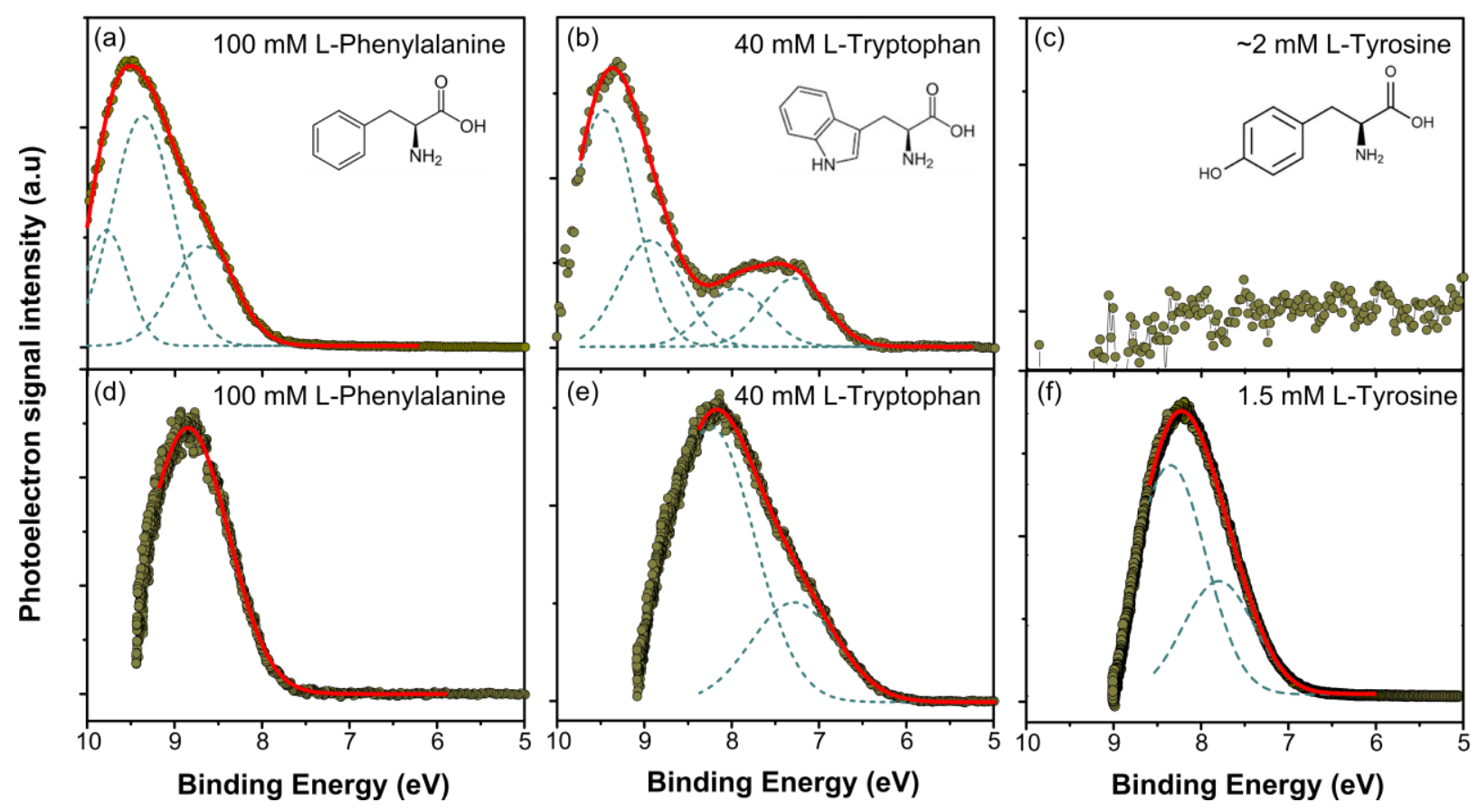

Figure 2: X-ray photoemission (a-c) and $267 \mathrm{~nm}$ resonant two photon ionization (d-f) spectra of the aromatic amino acids in water (solid circles). Individual Gaussian fits and the total fit are shown in blue dashed lines and solid red lines, respectively. Note that the R2PI spectra are affected by the instrument cutoff function at $\mathrm{eKE}<0.5 \mathrm{eV}$.
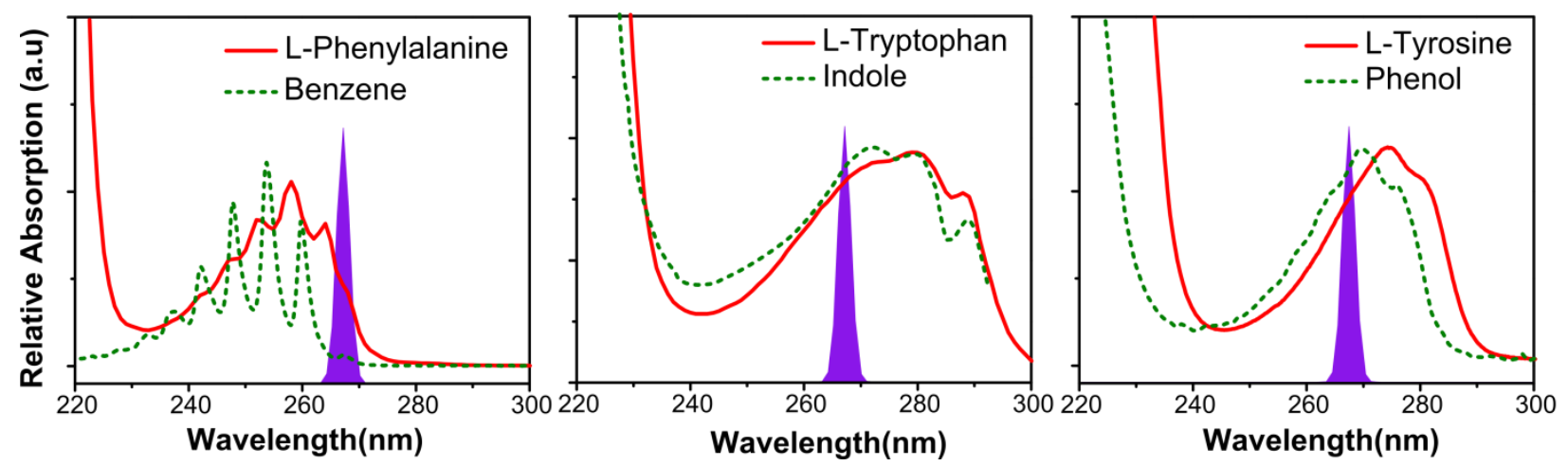

Figure 3: UV-VIS absorption spectra of aromatic amino acids in water. For comparison absorption spectra of benzene, indole and phenol in water are also shown. The solid purple band shows the excitation beam spectrum centered at $267 \mathrm{~nm}(\mathrm{FWHM} 3 \mathrm{~nm})$. 

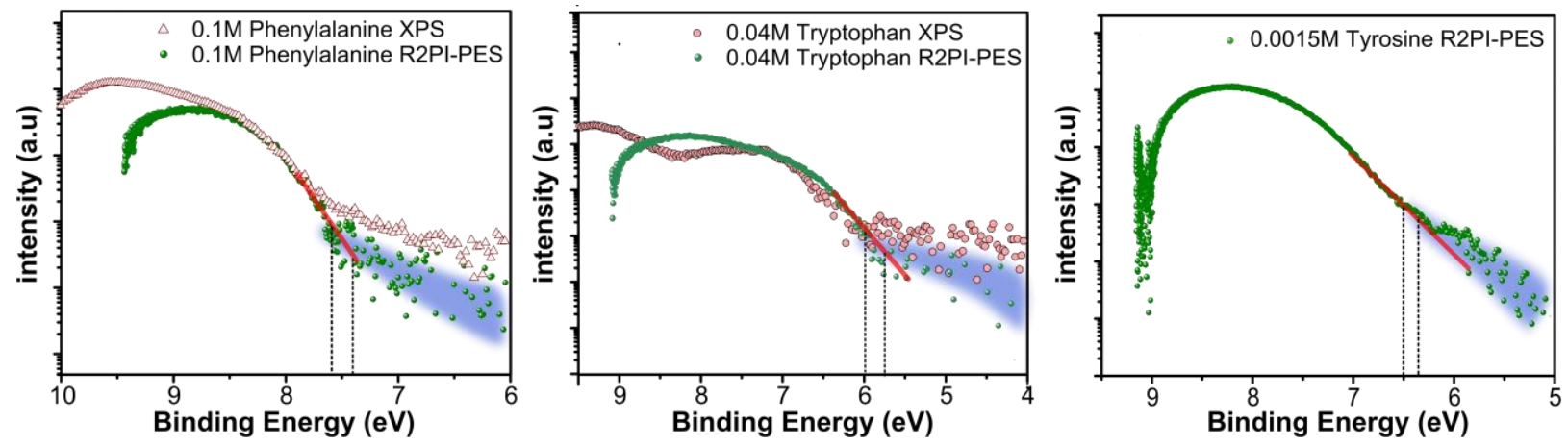

Figure 4: Estimation of the adiabatic ionization energies of aromatic amino acids. The intensity axis is plotted on a logarithmic scale to emphasize the noise floor. The red line indicates the rising edge of the signal and the blue region denotes the spread in estimated noise levels (see text). Due to the reference subtraction, the dynamic range in the signal intensity is low $(<400: 1)$ in XPS compared to R2PI measurements (>2000:1), which makes the assignment of the baseline more ambiguous in the XPS data.
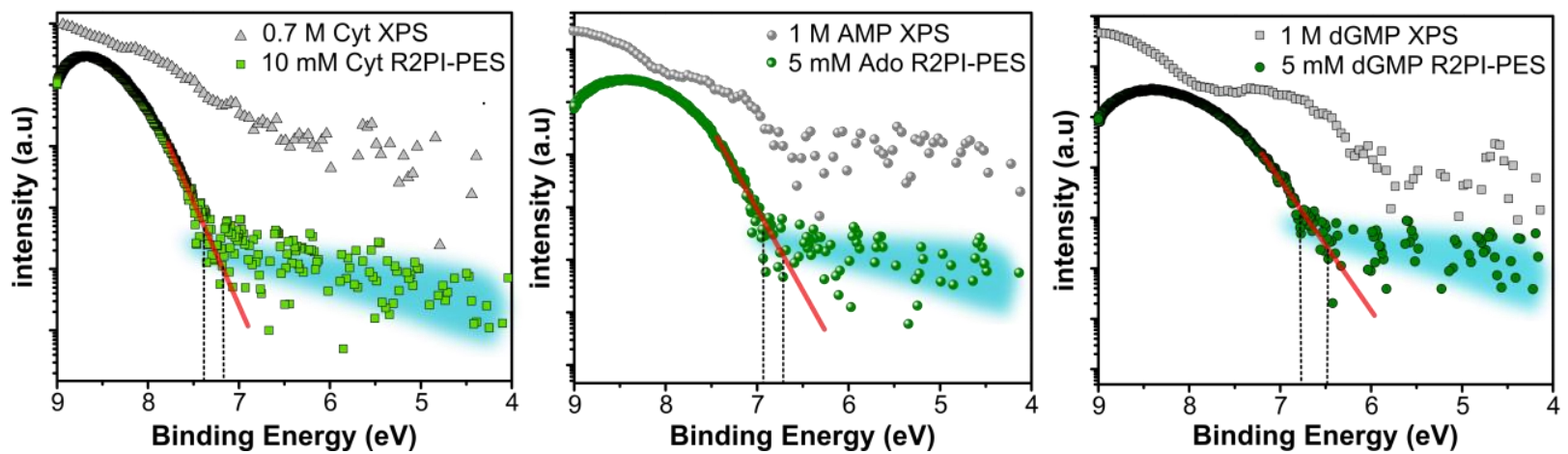

Figure 5: XPS and R2PI-PES spectra of cytidine (Cyt), adenosine (Ado) and deoxyguanosine monophosphate (dGMP) in water. AIEs have been estimated as described in the text. We find that AIE derived from these measurements overestimate the standard oxidation potential by $\sim 0.6 \mathrm{~V}( \pm 0.2 \mathrm{~V})$ when compared to the values reported in ref 14 ( $c f$. Table 2). See text for explanation. 
(1)

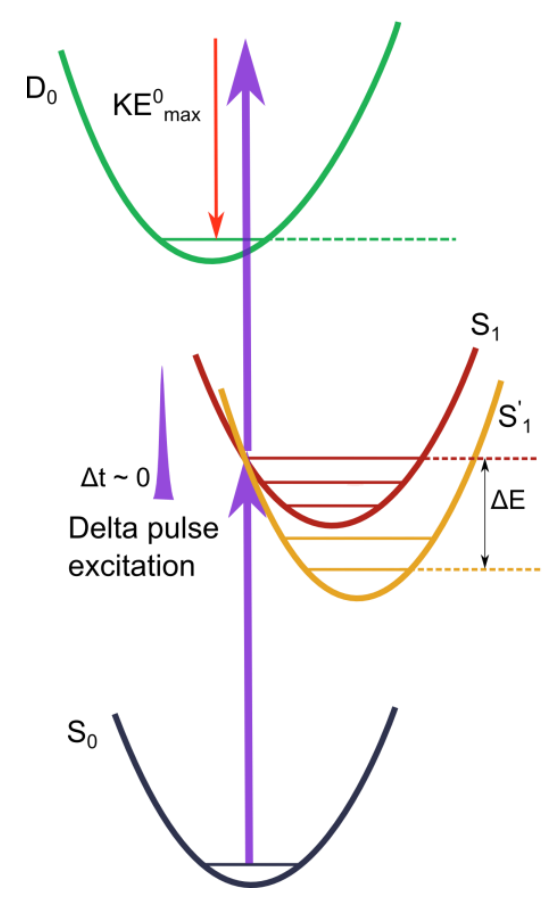

(2)

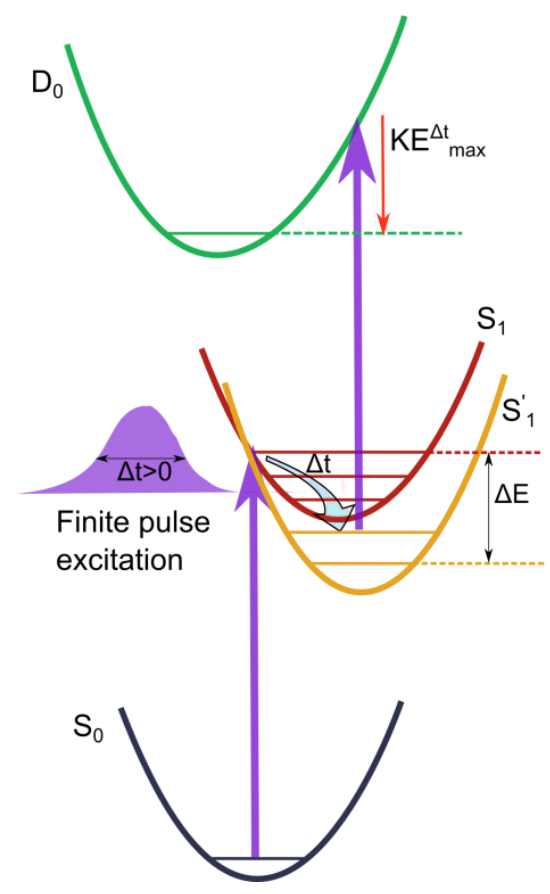

Figure 6: Schematic representation of the resonant two photon ionization process. With delta pulse excitation (case 1), the initial wave packet launched on the $S_{1}$ surface (FC region) undergoes ionization prior to any nuclear and electronic relaxation and reaches the final state $\mathrm{D}_{0}$ (radical cation). The excess energy is manifested as the electron kinetic energy. The maximum available kinetic energy corresponding to $\mathrm{S}_{0}(\mathrm{v}=0) \rightarrow \mathrm{D}_{0}(\mathrm{v}=0)$ transition is shown as $\mathrm{KE}^{0}{ }_{\text {max }}$. The adiabatic ionization energy can be defined as $\operatorname{AIE}(0)=2^{*} h v-\mathrm{KE}_{\text {max }}^{0}$. In case of a finite laser pulse (case 2 ), relaxation takes place within the pulse width $(\Delta \mathrm{t})$. The observed adiabatic ionization energy is $\mathrm{AIE}(\Delta \mathrm{t})=2^{*} \mathrm{~h} v-\mathrm{KE}^{\Delta \mathrm{t}}{ }_{\max }$. Since $\mathrm{KE}^{0}{ }_{\max } \geq \mathrm{KE}^{\Delta \mathrm{t}}{ }_{\max }$, the observed ionization threshold for case 2 will always be equal to or higher than the actual value (no relaxation, case 1). 
Table 1. Vertical ionization energies of the aromatic amino acids in aqueous solutions compared with gas phase

\begin{tabular}{|c|c|c|c|}
\hline Amino Acids & VIE $(e V) / X P S{ }^{a}$ & VIE (eV)/R2PI-PES a & VIE (eV)(gas phase) ${ }^{\mathbf{b}}$ \\
\hline L-Phenylalanine & $8.7\left(\pi_{3}\right), 9.4\left(\mathrm{n}_{N}\right), 9.8\left(\pi_{2}\right)$ & $8.8\left(\pi_{3}\right)$ & $8.9\left(\pi_{3}\right), 9.3\left(\mathrm{n}_{N}\right), 9.7\left(\pi_{2}\right)$ \\
\hline L-Tryptophan & $7.3\left(\pi_{3}\right), 8.0\left(\pi_{2}\right), 8.9\left(\mathrm{n}_{\mathrm{N}}\right), 9.5\left(\mathrm{n}_{\mathrm{N}}\right)$ & $7.3\left(\pi_{3}\right), 8.2\left(\pi_{2}\right)$ & $7.9\left(\pi_{3}\right), 8.3\left(\pi_{2}\right), 9.4\left(\mathrm{n}_{\mathrm{N}}\right), 9.8\left(\mathrm{n}_{\mathrm{N}}\right)$ \\
\hline L-Tyrosine & - & $7.8\left(\pi_{3}\right), 8.4\left(\pi_{2}\right)$ & $8.5\left(\pi_{3}\right), 9.4\left(\pi_{2}\right), 9.6\left(\mathrm{n}_{\mathrm{N}}\right)$ \\
\hline
\end{tabular}

${ }^{\text {a }}$ The standard error of VIE is $\pm 0.1 \mathrm{eV}$. Average FWHM of the photoelectron bands in solutions is $0.9 \pm 0.1 \mathrm{eV}$.

${ }^{\mathrm{b}}$ Gas phase values from refs. ${ }^{34-37}$

Table 2. Adiabatic ionization energies (in eV) and standard one electron reduction potentials (vs SHE, in V for couple given) for important biomolecules in aqueous solution.

\begin{tabular}{|c|c|c|c|c|c|c|}
\hline & $\mathrm{AIE}^{\mathrm{a}}$ & $\begin{array}{c}\mathbf{E}^{0}{ }_{\text {R2PI-PES }} \\
\left(A^{+\bullet / A}\right)^{b} \\
\text { vS SHE }\end{array}$ & $\begin{array}{c}\mathbf{E}^{0}\left(\mathbf{A}^{+\bullet} / \mathbf{A}\right) \\
\text { vs SHE }\end{array}$ & $\begin{array}{c}\mathrm{E}_{7}^{\mathrm{e}} \\
\text { vs } \mathrm{SHE}\end{array}$ & $\begin{array}{c}\mathrm{E}_{2}^{\mathbf{j}} \\
\text { vs SHE }\end{array}$ & $\begin{array}{c}\text { Reorganization } \\
\text { energy }(\lambda)^{h}\end{array}$ \\
\hline L-Phenylalanine & 7.5 & 3.22 & 3.2 & - & - & 1.3 \\
\hline L-Tryptophan & 5.9 & 1.62 & 1.6 & $1.015^{\mathrm{f}}$ & $1.15^{\mathrm{f}}$ & 1.4 \\
\hline L-Tyrosine & 6.4 & 2.12 & - & $0.93^{f}$ & $1.22^{\mathrm{f}}$ & 1.4 \\
\hline Cytidine & 7.3 & 3.02 & $2.4^{\mathrm{c}}, 2.14^{\mathrm{d}}$ & $\sim 1.6^{\mathrm{g}}$ & - & $1.5^{\mathrm{g}}$ \\
\hline Adenosine & 6.8 & 2.52 & $2.1^{\mathrm{c}}, 1.96^{\mathrm{d}}$ & $1.44^{\mathrm{g}}$ & - & $1.4^{\mathrm{g}}$ \\
\hline $\begin{array}{l}\text { Deoxyguanosine } \\
\text { monophosphate }\end{array}$ & 6.6 & 2.32 & $1.5^{\mathrm{c}}, 1.49^{\mathrm{d}}$ & $1.31^{\mathrm{g}}$ & - & $1.5^{\mathrm{g}}$ \\
\hline
\end{tabular}

${ }^{a}$ AIEs referenced to vacuum extracted from R2PI-PES experiments in solution (see text for details).

Errors associated with AIEs are $\pm 0.1 \mathrm{~V}$ for Phenylalanine and Tryptophan, and less than $\pm 0.1 \mathrm{~V}$ for Tyrosine (see Figure 4).

${ }^{\mathrm{b}} \mathrm{E}_{\mathrm{R} 2 \mathrm{PI}-\mathrm{PES}}^{0}=\mathrm{AIE}-\mathrm{E}_{\mathrm{SHE}}^{0}$ where $\mathrm{E}_{\mathrm{SHE}}^{0}=4.28 \mathrm{~V}$ from Refs. ${ }^{13,54}$. We note that the uncertainty of $\mathrm{E}_{\mathrm{SHE}}^{0}$ values in the literature is $\pm 0.2 \mathrm{~V}$. Refs. ${ }^{13,53-54}$. However, this uncertainty is common for all systems and will not change the relative values of the standard redox potential.

${ }^{\mathrm{c}} \mathrm{E}^{0}\left(\mathrm{~A}^{+\bullet} / \mathrm{A}\right)$ values from X-ray photoemission measurements ( $c f$. Table 4 in ref $\left.{ }^{14}\right)$.

${ }^{\mathrm{d}} \mathrm{E}^{0}\left(\mathrm{~A}^{+\bullet / A}\right)$ values in acetonitrile. Refs. ${ }^{14,51}$

${ }^{\mathrm{e}} \mathrm{E}_{7}$, one-electron reduction potential measured at $\mathrm{pH}=7$

${ }^{\mathrm{f}}$ from ref ${ }^{10}$, values are measured vs SHE

$\mathrm{g}_{\text {from ref }}{ }^{14}$

${ }^{h}$ Reorganization energy is derived from the R2PI VIEs in Table 1 (column 3) and AIEs here (column 2) as follows

$\lambda=\mathrm{VIE}_{\text {R2PI-PES }}-\mathrm{AIE}$

${ }^{\mathrm{j}} \mathrm{E}_{2}$, one-electron reduction potential measured at $\mathrm{pH}=2$ 


\section{References:}

- $\underline{1}$.

Westerlund, K.; Berry, B. W.; Privett, H. K.; Tommos, C. Exploring amino-acid radical chemistry: protein engineering and de novo design. Biochim. Biophys. Acta, Bioenerg. 2005, 1707 (1), 103-116, DOI: 10.1016/j.bbabio.2004.02.013

- $\underline{2}$.

Kovacic, P. Protein electron transfer mechanism and reproductive toxicity: Iminium, hydrogen bonding, homoconjugation, amino acid side chains redox and charged and cell signaling. Birth Defects Res., Part $C$ 2007, 81 (1), 51-64, DOI: 10.1002/bdrc.20086

\section{- $\underline{3}$.}

Bogdanov, A. M.; Mishin, A. S.; Yampolsky, I. V.; Belousov, V. V.; Chudakov, D. M.; Subach, F. V.; Verkhusha, V. V.; Lukyanov, S.; Lukyanov, K. A. Green fluorescent proteins are light-induced electron donors. Nat. Chem. Biol. 2009, 5 (7), 459-461, DOI: 10.1038/nchembio.174

- $\underline{4}$.

Winkler, J. R.; Gray, H. B. Could tyrosine and tryptophan serve multiple roles in biological redox processes?. Philos. Trans. R. Soc., A 2015, 373, 2037, DOI: 10.1098/rsta.2014.0178

\section{- $\underline{5}$.}

Morozova, O. B.; Kiryutin, A. S.; Sagdeev, R. Z.; Yurkovskaya, A. V. Electron Transfer between Guanosine Radical and Amino Acids in Aqueous Solution. 1. Reduction of Guanosine Radical by Tyrosine. J. Phys. Chem. B 2007, 111 (25), 7439- 7448, DOI: 10.1021/jp067722i

- $\underline{6}$.

Morozova, O. B.; Kiryutin, A. S.; Yurkovskaya, A. V. Electron Transfer between Guanosine Radicals and Amino Acids in Aqueous Solution. II. Reduction of Guanosine Radicals by Tryptophan. J. Phys. Chem. $B$ 2008, 112 (9), 2747- 2754, DOI: 10.1021/jp0752318

\section{- $\underline{7}$.}

Schlag, E. W.; Yang, D. Y.; Sheu, S. Y.; Selzle, H. L.; Lin, S. H.; Rentzepis, P. M. Dynamical principles in biological processes: A model of charge migration in proteins and DNA. Proc. Natl. Acad. Sci. U. S. A. 2000, 97 (18), 9849-9854, DOI: 10.1073/pnas.140196597

- $\underline{8}$. 
Marcus, R. A. On the Theory of Oxidation-Reduction Reactions Involving Electron Transfer. I. J. Chem.

Phys. 1956, 24 (5), 966- 978, DOI: 10.1063/1.1742723

- $\underline{9}$.

Marcus, R. A. On the Theory of Oxidation-Reduction Reactions Involving Electron Transfer. III. Applications to Data on the Rates of Organic Redox Reactions. J. Chem. Phys. 1957, 26 (4), 872- 877, DOI: $10.1063 / 1.1743424$

- $\underline{10}$.

Harriman, A. Further comments on the redox potentials of tryptophan and tyrosine. J. Phys. Chem. 1987, 91 (24), 6102-6104, DOI: 10.1021/j100308a011

- 11.

Jovanovic, S. V.; Harriman, A.; Simic, M. G. Electron-transfer reactions of tryptophan and tyrosine derivatives. J. Phys. Chem. 1986, 90 (9), 1935-1939, DOI: 10.1021/j100400a039

- $\underline{12}$.

Butler, J.; Land, E. J.; Swallow, A. J.; Prutz, W. A. Comment on "Electron-transfer reactions of tryptophan and tyrosine derivatives". J. Phys. Chem. 1987, 91 (11), 3113-3114, DOI: 10.1021/j100295a094

- $\underline{13}$.

Ghosh, D.; Roy, A.; Seidel, R.; Winter, B.; Bradforth, S. E.; Krylov, A. I. First-Principle Protocol for Calculating lonization Energies and Redox Potentials of Solvated Molecules and lons: Theory and Application to Aqueous Phenol and Phenolate. J. Phys. Chem. B 2012, 116 (24), 7269- 7280, DOI: 10.1021/jp301925k

- 14.

Schroeder, C. A.; Pluhařová, E.; Seidel, R.; Schroeder, W. P.; Faubel, M.; Slavíček, P.; Winter, B.; Jungwirth, P.; Bradforth, S. E. Oxidation Half-Reaction of Aqueous Nucleosides and Nucleotides via Photoelectron Spectroscopy Augmented by ab Initio Calculations. J. Am. Chem. Soc. 2015, 137 (1), 201209, DOI: 10.1021/ja508149e

- $\underline{15}$.

Wardman, P. Reduction potentials of one-electron couples involving free radicals in aqueous solution. J. Phys. Chem. Ref. Data 1989, 18 (4), 1637-1755, DOI: 10.1063/1.555843

- $\underline{16}$. 
Chatterley, A. S.; Johns, A. S.; Stavros, V. G.; Verlet, J. R. R. Base-Specific lonization of Deprotonated Nucleotides by Resonance Enhanced Two-Photon Detachment. J. Phys. Chem. A 2013, 117 (25), 52995305, DOI: 10.1021/jp4041315

- $\underline{17}$.

Ottosson, N.; Faubel, M.; Bradforth, S. E.; Jungwirth, P.; Winter, B. Photoelectron spectroscopy of liquid water and aqueous solution: Electron effective attenuation lengths and emission-angle anisotropy. J. Electron Spectrosc. Relat. Phenom. 2010, 177 (2-3), 60- 70, DOI: 10.1016/j.elspec.2009.08.007

- 18.

Thürmer, S.; Seidel, R.; Faubel, M.; Eberhardt, W.; Hemminger, J. C.; Bradforth, S. E.; Winter, B. Photoelectron Angular Distributions from Liquid Water: Effects of Electron Scattering. Phys. Rev. Lett. 2013, 111 (17), 173005, DOI: 10.1103/PhysRevLett.111.173005

- $\underline{19}$.

Suzuki, Y.-I.; Nishizawa, K.; Kurahashi, N.; Suzuki, T. Effective attenuation length of an electron in liquid water between 10 and 600 eV. Phys. Rev. E 2014, 90 (1), 010302, DOI: 10.1103/PhysRevE.90.010302

- $\underline{20}$.

Buchner, F.; Schultz, T.; Lubcke, A. Solvated electrons at the water-air interface: surface versus bulk signal in low kinetic energy photoelectron spectroscopy. Phys. Chem. Chem. Phys. 2012, 14 (16), 58375842, DOI: $10.1039 /$ c2cp23305c

- $\underline{21}$.

Winter, B.; Faubel, M. Photoemission from liquid aqueous solutions. Chem. Rev. 2006, 106 (4), 11761211, DOI: $10.1021 / \mathrm{cr040381p}$

- $\underline{22}$.

Faubel, M.; Schlemmer, S.; Toennies, J. P. A molecular beam study of the evaporation of water from a liquid jet. Z. Phys. D: At., Mol. Clusters 1988, 10, 269, DOI: 10.1007/BF01384861

\section{- 23.}

Dragonmir, A.; McInerney, J. G.; Nikogosyan, D. N. Femtosecond measurements of two-photon absorption coefficients at lambda $=264 \mathrm{~nm}$ in glasses, crystals, and liquids. Appl. Opt. 2002, 41 (21), 4365-4376, DOI: 10.1364/AO.41.004365

- 24. 
Valadan, M.; Davide, D. A.; Felice, G.; Raffaele, V.; Carlo, A. Temporal and spectral characterization of femtosecond deep-UV chirped pulses. Laser Phys. Lett. 2015, 12 (2), 025302, DOI: 10.1088/16122011/12/2/025302

- 25.

Kruit, P.; Read, F. H. Magnetic field paralleliser for $2 \pi$ electron-spectrometer and electron-image magnifier. J. Phys. E: Sci. Instrum. 1983, 16 (4), 313, DOI: 10.1088/0022-3735/16/4/016

- $\underline{26}$.

Rijs, A. M.; Backus, E. H. G.; de Lange, C. A.; Westwood, N. P. C.; Janssen, M. H. M. 'Magnetic bottle' spectrometer as a versatile tool for laser photoelectron spectroscopy. J. Electron Spectrosc. Relat. Phenom. 2000, 112 (1-3), 151- 162, DOI: 10.1016/S0368-2048(00)00209-7

- $\underline{27}$.

Buchner, F.; Lübcke, A.; Heine, N.; Schultz, T. Time-resolved photoelectron spectroscopy of liquids. Rev. Sci. Instrum. 2010, 81 (11), 113107, DOI: 10.1063/1.3499240

\section{- $\underline{28}$.}

Tang, Y.; Shen, H.; Sekiguchi, K.; Kurahashi, N.; Mizuno, T.; Suzuki, Y. I.; Suzuki, T. Direct measurement of vertical binding energy of a hydrated electron. Phys. Chem. Chem. Phys. 2010, 12 (15), 3653- 3655, DOI: $10.1039 / \mathrm{b} 925741 \mathrm{a}$

\section{- $\underline{29}$.}

Shen, H. A.; Kurahashi, N.; Horio, T.; Sekiguchi, K.; Suzuki, T. Direct Measurement of Vertical Electron Binding Energies of Solvated Electrons in Methanol and Ethanol. Chem. Lett. 2010, 39 (7), 668- 670, DOI: $10.1246 / \mathrm{cl} .2010 .668$

\section{- $\underline{30}$.}

Preissler, N.; Buchner, F.; Schultz, T.; Lübcke, A. Electrokinetic Charging and Evidence for Charge Evaporation in Liquid Microjets of Aqueous Salt Solution. J. Phys. Chem. B 2013, 117 (8), 2422- 2428, DOI: $10.1021 / \mathrm{jp} 304773 \mathrm{n}$

\section{- $\underline{31}$.}

Shreve, A. T.; Elkins, M. H.; Neumark, D. M. Photoelectron spectroscopy of solvated electrons in alcohol and acetonitrile microjets. Chemical Science 2013, 4 (4), 1633-1639, DOI: 10.1039/c3sc22063j

\section{- $\underline{32}$.}

Kurahashi, N.; Karashima, S.; Tang, Y.; Horio, T.; Abulimiti, B.; Suzuki, Y.-I.; Ogi, Y.; Oura, M.; Suzuki, T. Photoelectron spectroscopy of aqueous solutions: Streaming potentials of $\mathrm{NaX}(\mathrm{X}=\mathrm{Cl}, \mathrm{Br}$, and $\mathrm{I})$ 
solutions and electron binding energies of liquid water and X-. J. Chem. Phys. 2014, 140 (17), 174506, DOI: $10.1063 / 1.4871877$

\section{- $\underline{33}$.}

Elles, C. G.; Rivera, C.; Zhang, X.; Pieniazek, P. A.; Bradforth, S. E. Electronic structure of liquid water from polarization-dependent two-photon absorption spectroscopy. J. Chem. Phys. 2009, 130, 084501, DOI: 10.1063/1.3078336

\section{- $\underline{34}$.}

Cannington, P. H.; Ham, N. S. He(I) and He(II) photoelectron spectra of glycine and related molecules. J. Electron Spectrosc. Relat. Phenom. 1983, 32 (2), 139-151, DOI: 10.1016/0368-2048(83)85092-0

- 35.

Campbell, S.; Beauchamp, J. L.; Rempe, M.; Lichtenberger, D. L. Correlations of lone pair ionization energies with proton affinities of amino acids and related compounds. Site specificity of protonation. Int. J. Mass Spectrom. Ion Processes 1992, 117 (0), 83- 99, DOI: 10.1016/0168-1176(92)80087-H

\section{- $\underline{36}$.}

Campbell, S.; Marzluff, E. M.; Rodgers, M. T.; Beauchamp, J. L.; Rempe, M. E.; Schwinck, K. F.; Lichtenberger, D. L. Proton Affinities and Photoelectron Spectra of Phenylalanine and N-Methyl- and $\mathrm{N}, \mathrm{N}$-Dimethylphenylalanine. Correlation of Lone Pair lonization Energies with Proton Affinities and Implications for N-Methylation as a Method to Effect Site Specific Protonation of Peptides. J. Am. Chem. Soc. 1994, 116 (12), 5257- 5264, DOI: 10.1021/ja00091a033

\section{- $\underline{37}$.}

Seki, K.; Inokuchi, H. Photoelectron spectrum of I-tryptophan in the gas phase. Chem. Phys. Lett. 1979, 65 (1), 158-160, DOI: 10.1016/0009-2614(79)80148-7

\section{- $\underline{38}$.}

Plekan, O.; Feyer, V.; Richter, R.; Coreno, M.; Prince, K. C. Valence photoionization and photofragmentation of aromatic amino acids. Mol. Phys. 2008, 106 (9-10), 1143-1153, DOI: $10.1080 / 00268970801974875$

\section{- $\underline{39}$.}

Dehareng, D.; Dive, G. Vertical lonization Energies of $\alpha$-L-Amino Acids as a Function of Their Conformation: an Ab Initio Study. Int. J. Mol. Sci. 2004, 5 (11), 301-332, DOI: 10.3390/i5110301

- $\underline{40}$. 
Winter, B.; Weber, R.; Hertel, I. V.; Faubel, M.; Jungwirth, P.; Brown, E. C.; Bradforth, S. E. Electron binding energies of aqueous alkali and halide ions: EUV photoelectron spectroscopy of liquid solutions and combined ab initio and molecular dynamics calculations. J. Am. Chem. Soc. 2005, 127 (19), 72037214, DOI: 10.1021/ja042908I

\section{- $\underline{41}$.}

Jagoda-Cwiklik, B.; Slaviek, P.; Cwiklik, L.; Nolting, D.; Winter, B.; Jungwirth, P. Ionization of imidazole in the gas phase, microhydrated environments, and in aqueous solution. J. Phys. Chem. A 2008, 112 (16), 3499-3505, DOI: 10.1021/jp711476g

\section{- $\underline{42}$.}

Platt, J. R. Classification of Spectra of Cata-Condensed Hydrocarbons. J. Chem. Phys. 1949, 17, 484, DOI: 10.1063/1.1747293

- 43.

Murrell, J. N. The Theory of the Electronic Spectra of Organic Molecules; Chapman and Hall: London, 1971.

- $\underline{44}$.

Weber, G. Fluorescence-Polarization Spectrum and Electronic-Energy Transfer in Tyrosine, Tryptophan and related Compounds. Biochem. J. 1960, 75, 335, DOI: 10.1042/bj0750335

\section{- $\underline{45}$.}

Serrano-Andrés, L.; Roos, B. O. Theoretical Study of the Absorption and Emission Spectra of Indole in the Gas Phase and in a Solvent. J. Am. Chem. Soc. 1996, 118 (1), 185- 195, DOI: 10.1021/ja952035i

\section{- $\underline{46}$.}

Dixon, R. N.; Oliver, T. A. A.; Ashfold, M. N. R. Tunnelling under a conical intersection: Application to the product vibrational state distributions in the UV photodissociation of phenols. J. Chem. Phys. 2011, 134 (19), 194303, DOI: 10.1063/1.3585609

\section{- $\underline{47}$.}

Schick, C. P.; Carpenter, S. D.; Weber, P. M. Femtosecond multiphoton ionization photoelectron spectroscopy of the S-2 state of phenol. J. Phys. Chem. A 1999, 103 (49), 10470-10476, DOI: 10.1021/jp992065y

\section{- $\underline{48}$.}


Buchner, F.; Ritze, H.-H.; Lahl, J.; Lubcke, A. Time-resolved photoelectron spectroscopy of adenine and adenosine in aqueous solution. Phys. Chem. Chem. Phys. 2013, 15 (27), 11402-11408, DOI:

$10.1039 / \mathrm{c3cp51057c}$

- $\underline{49}$.

Adriaanse, C.; Sulpizi, M.; VandeVondele, J.; Sprik, M. The electron attachment energy of the aqueous hydroxyl radical predicted from the detachment energy of the aqueous hydroxide anion. J. Am. Chem. Soc. 2009, 131 (17), 6046- 7, DOI: 10.1021/ja809155k

- $\underline{50}$.

Rubesova, M.; Muchova, E.; Slavicek, P. Optimal Tuning of Range-Separated Hybrids for Solvated Molecules with Time-Dependent Density Functional Theory. J. Chem. Theory Comput. 2017, 13 (10), 4972-4983, DOI: 10.1021/acs.jctc.7b00675

- $\underline{51}$.

Seidel, C. A.; Schulz, A.; Sauer, M. H. Nucleobase-Specific Quenching of Fluorescent Dyes. 1. Nucleobase One-Electron Redox Potentials and Their Correlation with Static and Dynamic Quenching Efficiencies. J. Phys. Chem. 1996, 100, 5541, DOI: 10.1021/jp951507c

\section{- $\underline{52}$.}

Steenken, S. Purine bases, nucleosides, and nucleotides: aqueous solution redox chemistry and transformation reactions of their radical cations and e- and $\mathrm{OH}$ adducts. Chem. Rev. 1989, 89 (3), 503520, DOI: 10.1021/cr00093a003

\section{- $\underline{53}$.}

Truhlar, D. G.; Cramer, C. J. Molecular Modeling of Environmentally Important Processes: Reduction Potentials. J. Chem. Educ. 2004, 81, 596, DOI: 10.1021/ed081p596

\section{- $\underline{54}$.}

Isse, A. A.; Gennaro, A. Absolute Potential of the Standard Hydrogen Electrode and the Problem of Interconversion of Potentials in Different Solvents. J. Phys. Chem. B 2010, 114, 7894, DOI:

10.1021/jp100402x

\section{- $\underline{55}$.}

Ullrich, S.; Schultz, T.; Zgierski, M. Z.; Stolow, A. Direct Observation of Electronic Relaxation Dynamics in Adenine via Time-Resolved Photoelectron Spectroscopy. J. Am. Chem. Soc. 2004, 126, 2262- 2263, DOI: 10.1021/ja030532q

- $\underline{56}$. 
Ullrich, S.; Schultz, T.; Zgierski, M. Z.; Stolow, A. Electronic relaxation dynamics in DNA and RNA bases studied by time-resolved photoelectron spectroscopy. Phys. Chem. Chem. Phys. 2004, 6, 2796- 2801, DOI: 10.1039/b316324e

- $\underline{57}$.

Jailaubekov, A. Ph.D. Thesis, University of Southern California, Los Angeles, CA, 2007.

- $\underline{58}$.

Yamazaki, S.; Kato, S. Solvent Effect on Conical Intersections in Excited-State 9H-Adenine: Radiationless Decay Mechanism in Polar Solvent. J. Am. Chem. Soc. 2007, 129 (10), 2901- 2909, DOI:

10.1021/ja0669169

- $\underline{59}$.

Karunakaran, V.; Kleinermanns, K.; Improta, R.; Kovalenko, S. A. Photoinduced Dynamics of Guanosine Monophosphate in Water from Broad-Band Transient Absorption Spectroscopy and Quantum-Chemical Calculations. J. Am. Chem. Soc. 2009, 131 (16), 5839- 5850, DOI: 10.1021/ja810092k

- 60.

Miannay, F.-A.; Gustavsson, T.; Banyasz, A.; Markovitsi, D. Excited-State Dynamics of dGMP Measured by Steady-State and Femtosecond Fluorescence Spectroscopy. J. Phys. Chem. A 2010, 114 (9), 3256- 3263, DOI: $10.1021 /$ jp909410b

- $\underline{61}$.

Barbatti, M.; Aquino, A. J. A.; Szymczak, J. J.; Nachtigallová, D.; Hobza, P.; Lischka, H. Relaxation mechanisms of UV-photoexcited DNA and RNA nucleobases. Proc. Natl. Acad. Sci. U. S. A. 2010, 107 (50), 21453-21458, DOI: 10.1073/pnas.1014982107

- 62.

Bräm, O.; Oskouei, A. A.; Tortschanoff, A.; van Mourik, F.; Madrid, M.; Echave, J.; Cannizzo, A.; Chergui, M. Relaxation Dynamics of Tryptophan in Water: A UV Fluorescence Up-Conversion and Molecular Dynamics Study. J. Phys. Chem. A 2010, 114 (34), 9034- 9042, DOI: 10.1021/jp101778u

- $\underline{63}$.

Leonard, J.; Sharma, D.; Szafarowicz, B.; Torgasin, K.; Haacke, S. Formation dynamics and nature of tryptophan's primary photoproduct in aqueous solution. Phys. Chem. Chem. Phys. 2010, 12 (48), 1574415750, DOI: 10.1039/c0cp00615g

- $\underline{64}$. 
Sharma, D.; Léonard, J.; Haacke, S. Ultrafast excited-state dynamics of tryptophan in water observed by transient absorption spectroscopy. Chem. Phys. Lett. 2010, 489 (1-3), 99- 102, DOI:

10.1016/j.cplett.2010.02.057

- $\underline{65}$.

Peon, J.; Hess, G. C.; Pecourt, J.-M. L.; Yuzawa, T.; Kohler, B. Ultrafast Photoionization Dynamics of Indole in Water. J. Phys. Chem. A 1999, 103 (14), 2460- 2466, DOI: 10.1021/jp9840782

- $\underline{66}$.

Pagba, C. V.; Chi, S.-H.; Perry, J.; Barry, B. A. Proton-Coupled Electron Transfer in Tyrosine and a $\beta$ Hairpin Maquette: Reaction Dynamics on the Picosecond Time Scale. J. Phys. Chem. B 2015, 119 (6), 2726- 2736, DOI: 10.1021/jp510171z

- $\underline{67}$.

Zhang, Y. Ph.D. Thesis, University of Southern California, Los Angeles, CA, 2007.

- $\underline{68}$.

Oliver, T. A. A.; Zhang, Y.; Roy, A.; Ashfold, M. N. R.; Bradforth, S. E. Exploring the autoionization and photo-induced proton-coupled electron transfer pathways of phenol in aqueous solution. J. Phys. Chem. Lett. 2015, 6 (20), 4159-4164, DOI: 10.1021/acs.jpclett.5b01861

- $\underline{69}$.

Luria, M.; Ofran, M.; Stein, G. Natural and experimental fluorescence lifetimes of benzene in various solvents. J. Phys. Chem. 1974, 78 (19), 1904-1909, DOI: 10.1021/j100612a005

- $\underline{70}$.

Lee, S.-H.; Tang, K.-C.; Chen, I. C.; Schmitt, M.; Shaffer, J. P.; Schultz, T.; Underwood, J. G.; Zgierski, M. Z.; Stolow, A. Substituent Effects in Molecular Electronic Relaxation Dynamics via Time-Resolved Photoelectron Spectroscopy: $\pi \pi^{*}$ States in Benzenes. J. Phys. Chem. A 2002, 106 (39), 8979- 8991, DOI: 10.1021/jp021096h

- $\underline{71}$.

Parker, D. S. N.; Minns, R. S.; Penfold, T. J.; Worth, G. A.; Fielding, H. H. Ultrafast dynamics of the S1 excited state of benzene. Chem. Phys. Lett. 2009, 469 (1-3), 43- 47, DOI: 10.1016/j.cplett.2008.12.069

- $\underline{72}$.

Das, T. N. Oxidation of Phenol in Aqueous Acid: Characterization and Reactions of Radical Cations vis-àvis the Phenoxyl Radical. J. Phys. Chem. A 2005, 109 (21), 3344-3351, DOI: 10.1021/jp050015p 
- $\underline{73}$.

Mainfray, G.; Manus, C. Resonance effects in multiphoton ionization of atoms. Appl. Opt. 1980, 19 (23), 3934- 3940, DOI: 10.1364/AO.19.003934

\section{- $\underline{74}$.}

Lompre, L. A.; Mainfray, G.; Manus, C. Multiphoton resonance effects in very high laser fields: twelvephoton ionisation of krypton at $10^{13} \mathrm{~W} \mathrm{~cm}^{-2}$. J. Phys. B: At. Mol. Phys. 1980, 13 (1), 85- 99, DOI:

10.1088/0022-3700/13/1/016

- $\underline{75}$.

Chatterley, A. S.; West, C. W.; Roberts, G. M.; Stavros, V. G.; Verlet, J. R. R. Mapping the Ultrafast Dynamics of Adenine onto Its Nucleotide and Oligonucleotides by Time-Resolved Photoelectron Imaging. J. Phys. Chem. Lett. 2014, 5 (5), 843-848, DOI: 10.1021/jz500264c

- $\underline{76}$.

Chatterley, A. S.; West, C. W.; Stavros, V. G.; Verlet, J. R. R, Time-resolved photoelectron imaging of the isolated deprotonated nucleotides. Chemical Science 2014, 5 (10), 3963-3975, DOI:

10.1039/C4SC01493F

- $\underline{77}$.

Yang, X.; Wang, X.-B.; Vorpagel, E. R.; Wang, L.-S. Direct Experimental Observation of the Low lonization Potentials of Guanine in Free Oligonucleotides by Using Photoelectron Spectroscopy. Proc. Natl. Acad. Sci. U. S. A. 2004, 101 (51), 17588-17592, DOI: 10.1073/pnas.0405157101

- $\underline{78}$.

Luckhaus, D.; Yamamoto, Y.; Suzuki, T.; Signorell, R. Genuine binding energy of the hydrated electron. Science Advances 2017, 3 (4), e1603224, DOI: 10.1126/sciadv.1603224

- $\underline{79}$.

Riley, J. W.; Wang, B.; Woodhouse, J. L.; Assmann, M.; Worth, G. A.; Fielding, H. H. Unravelling the Role of an Aqueous Environment on the Electronic Structure and Ionization of Phenol Using Photoelectron Spectroscopy. J. Phys. Chem. Lett. 2018, 9 (4), 678- 682, DOI: 10.1021/acs.jpclett.7b03310

- $\underline{80}$.

Chen, H.; Li, S. Ab initio study of deactivation pathways of excited 9H-guanine. J. Chem. Phys. 2006, 124 (15), 154315, DOI: 10.1063/1.2186998 
Table of Content Graphic

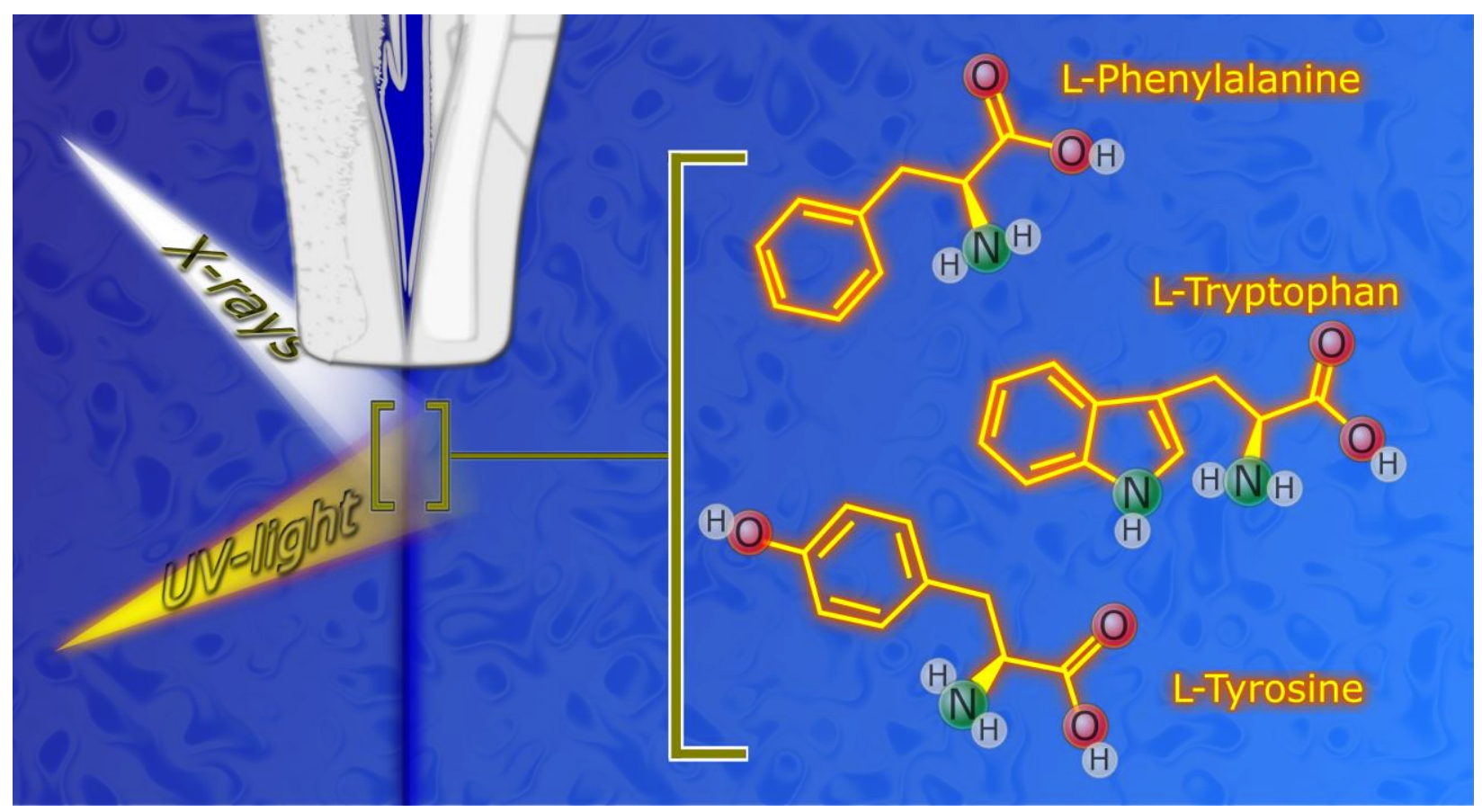

